\title{
Phytochemicals and the Consumer: Factors Affecting Fruit and Vegetable Consumption and the Potential for Increasing Small Fruit in the Diet
}

\author{
Joseph C. Scheerens ${ }^{1}$
}

Additional Index words. antioxidant, flavonoid, Food Guide Pyramid, 5 A Day-for Better Health program, food choice model, fruit quality, sensory perception

\begin{abstract}
Summary. Increasing fruit and vegetable consumption reduces risk factors for cancer, cardiovascular disease and a number of other diet-related chronic diseases. These foodstuffs contain relatively high levels of beneficial phytochemicals (plant-derived, biologically active compounds) among which the preventative activity of antioxidants are most well-known and well-documented. Since small fruit typically contain high levels of antioxidants, increasing their incorporation in the diet is a laudable goal. Media reports of medical studies pertaining to dietary intake and national education initiatives such as the USDA's Food Guide Pyramid and the 5 A Dayfor Better Health program have successfully raised public awareness of the health benefits of increased fruit and vegetable consumption, but, as of yet, may not have altered dietary habits. The factors influencing food choice are complex and interrelated. They include: sensory preference, physiological factors (pre- and postingestion), age, gender, lifestyle, behavior, personality, education, income, social attitudes about diet and health, ethnicity and tradition, religious beliefs, social pressures, marketing pressures, available product information and knowledge (labeling, media coverage, etc.) or self-identity beliefs. Some of these factors offer opportunities for increasing fruit and vegetable consumption while others present challenges. With respect to small fruit, food choice factors that tend to increase consumption include public awareness of these products as being beneficial to health and longevity and their image as highly desirable, dessert-like commodities with exquisite flavors. The main factors that deter increased small fruit consumption include their relatively high price per serving and their relative perishability which affects cost, ease of transport and availability. Strategies to capitalize on small fruits' positive attributes and overcome negative attributes with respect to food choice include the application of innovative marketing strategies at all levels and the expansion of research efforts to optimize the health benefits and sensory quality of these products.
\end{abstract}

The evidence for the potential health benefits of increased fruit and veg etable consumption is undeniably convincing. According to Van Duyn (1999), reduced risks for various types of cancer, heart disease, stroke,

hypertension, birth defects, cataracts, diabetes, diverticulosis, and obesity have been conclusively linked to the ingestion of phytochemicals (plant-derived, biologically active compounds) commonly found in these commodities. Important fruit and vegetable phytochemical components, potentially active against these degenerative diseases are diverse (Table 1 ).

Published as contribution HCS 01-1 of the Ohio Agricultural Research and Development Center.

${ }^{1}$ Associate professor, Department of Horticulture and Crop Science, The Ohio State University, The Ohio Agricultural Research and Development Center, 1680 Madison Ave., Wooster, OH 44691. 
Among phytochemical effects, the role of antioxidants, such as vitamin $\mathrm{E}$ $(\alpha$-tocopherol), vitamin $\mathrm{C}$ (ascorbic acid), carotenoids, phenolic acids, trihydroxystilbenes, and flavonoids, in the prevention of certain cancers and cardiovascular diseases is perhaps most well-known and accepted (Creasy and Creasy, 1998; Hertog et al., 1997; Leake, 1997; Jang and Pezzuto, 1999; Maas et al., 1991; Steinmetz and Potter, 1996). Most small fruit and grapes contain relatively high antioxidant (ORAC) activity (Häkkinen et al., 1999; Shahidi and Naczk, 1995; Wang et al., 1996) and therefore may serve as an excellent source of these compounds in the diet. In fact, the USDA recently characterized blueberries, blackberries, raspberries and strawberries and raisins as top antioxidant foods (USDAARS, 1999). Within this workshop,
Kalt et al. (2001) reviewed levels of antioxidants found in blueberries and other small fruit and outlined horticultural parameters that affect antioxidant content. Small fruit and grape products such as preserves, juices, and wine are also high in antioxidant activity (Creasy and Creasy, 1998; Leake, 1997; Rommel and Wrolstad, 1993; Scarlata and Ebeler, 1999; Wang et al., 1996).

Obviously, promoting increased levels of antioxidants and other beneficial phytochemicals in the average diet is a laudable goal for health and nutrition professionals and for the food production, processing, and distribution industries. In our fast food culture, persuading men, women and children to eat more vegetables, fruit and specifically berries and other small fruit rich in antioxidants is a complex and perhaps challenging task. The following information is an attempt to assess where we are in this process and to suggest, with respect to small fruit, ways in which we can improve chances for success. Perhaps the first two questions to ask would be Are the health benefits of increased fruit and vegetable consumption common knowledge among consumers? If so, are current levels of fruit and vegetable consumption adequate for maintaining health and nutritional balance?

\section{Public awareness and per capita consumption}

For as many as 40 years, nutritionists, teachers, parents, etc. extolled the virtues of fruit and vegetables as one of the four basic food groups and as such, part of a well-balanced diet (Achterberg, 1994). However, the

Table 1. Fruit and vegetable phytochemical components and reduced diet-related health risks ${ }^{\mathrm{z}}$.

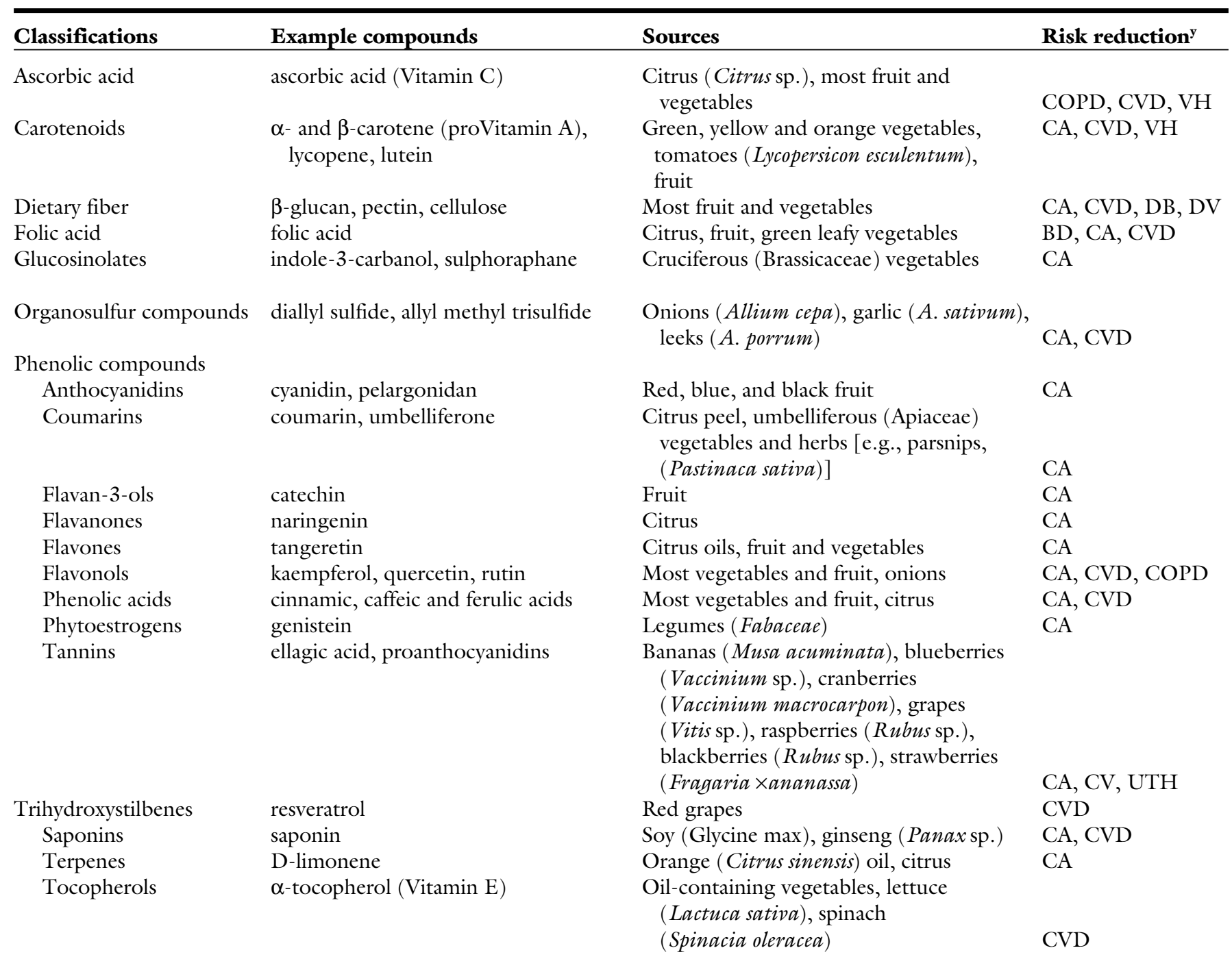

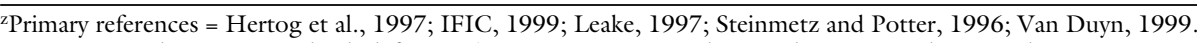

yegenerative diseases: $\mathrm{BD}=$ birth defects; $\mathrm{CA}=$ cancer; $\mathrm{COPD}=$ chronic obstructive pulmonary disease; CVD = cardiovascular disease including heart disease, stroke and hypertension; $\mathrm{DB}=$ diabetes mellitu; $\mathrm{DV}=$ diverticulosis; $\mathrm{UTH}=$ urinary tract health. 

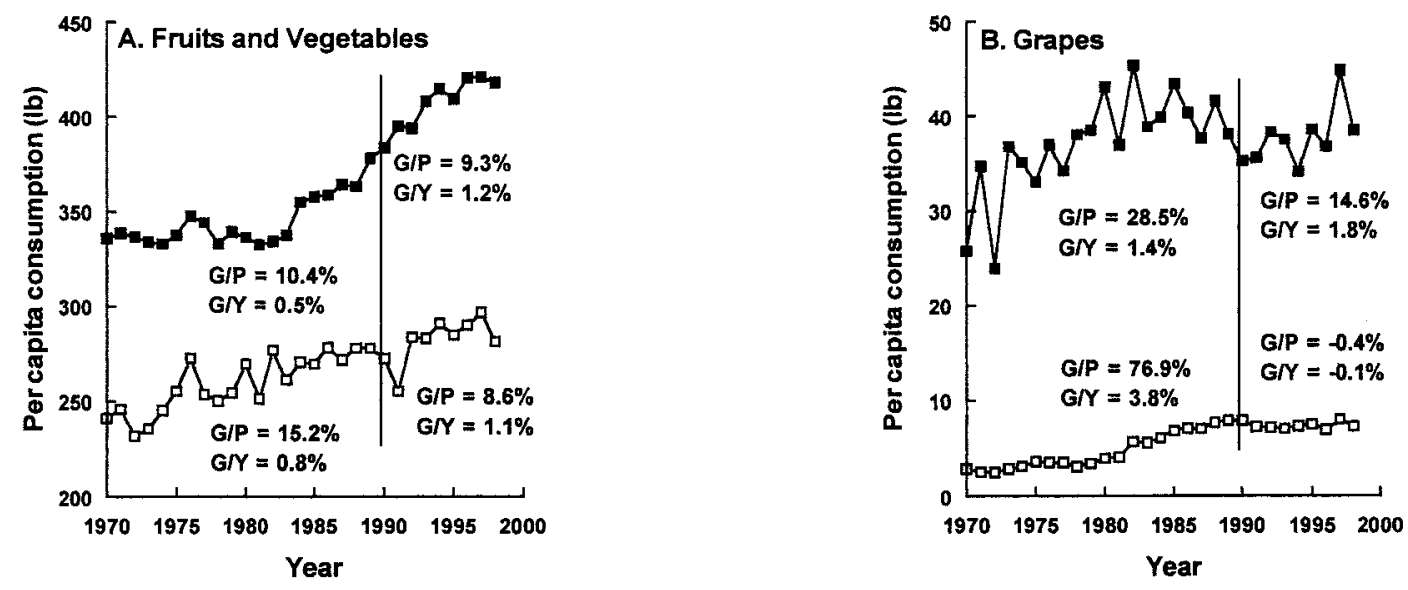

SecondNational Health and Nutrition Examination Survey (NHANES II, 1976$80)$ conducted by the National Center for Health, indicated from daily dietary recall that $45 \%$ of the respondents had no servings of fruit, $22 \%$ had no servings of vegetables and only $9 \%$ had the recommended servings of both (Patterson etal., 1990). In an attempt to improve these statistics, national education programs have been deployed such as those based on the USDA Food Guide Pyramid (Achterberg, 1994; Food Technology EditorialStaff, 1992, Chapman, 1999), designed to teach the nutritional parameters of variety, moderationand proportionality in terms of the total diet, and the national 5 A Day - for Better Health program, the nation's largest public/ private nutrition education program promoting increased fruit and vegetable consumption (Balch et al., 1997). Similar programs have been implemented by the World Health Organization (WHO) and other governmental agencies (Cox et al., 1998).

In addition toeducational initiatives, media coverage of medical research has heightened the public's awareness of the potential health benefits

Fig. 1. Per capita consumption in $1 \mathrm{bs}(1.00 \mathrm{lb}=0.454 \mathrm{~kg})$ of $(\mathrm{A})$ fruit and vegetables $(\square$ and $\square$, respectively) and (B-F) fresh and processed small fruit ( $\square$ and $\square$, respectively) from 1970-89 and from 1990-98. Percentage growth/period (G/P) and percentage annual growth within periods $(G / Y)$ are based on regression statistics calculated from tabular data of Putnam and Allshouse (1999). Graphs include data from 27 fresh fruit commodities and canned, frozen and dried fruit and fruit juices and from 25 fresh vegetable commodities and canned, frozen and dry or dried vegetables and chipping potatoes (A), fresh grapes canned and dried grapes (raisins), grape juice, and grapes used for wine making (B), fresh and frozen strawberries (C), fresh cranberries and cranberry juice (tabular data converted from gallons to pounds based an assumed density of 1.0 for raw cranberry juice) (D) frozen blackberries, raspberries and miscellaneous brambles (E) and frozen blueberries (F). Small fruit freezer stock sold retail or used in other food products. 
of improved diets, higher antioxidant intake and increased consumption of fruit and vegetables (Abbott, 1997; Farmakalidis, 1999; Wrick, 1995). Recent surveys by organizations such as the American Dietetic Association, the Food Marketing Institute, Health Focus 1999, the Leatherhead Research Group (UK) and the United Soybean Council suggested that $70 \%$ to $90 \%$ of consumers regard fruit and vegetables as very nutritious, health-conserving foods that are important constituents in a well-balanced diet (Farmakalidis, 1999; Sloan, 1993, 2000).

The success of educational initiatives and the effects of increased public awareness on health-diet issues can be assessed in several ways. Per capita consumption based on commodity disappearance data tabulated yearly by the USDA Economic Research Service (Putnam and Allshouse, 1999) offers one measure of change in the eating habits of the U.S. population. Figure 1 depicts U.S. per capita consumption patterns for fruit and vegetable commodities in general and for common small fruit and grape crops. Data are shown for two periods, 197089 (prefood guide pyramid) and 199098. Per capita consumption increases were calculated per period, both on a period and annual basis, based on regression statistics calculated from the tabular data of Putnam and Allshouse (1999).

Overall fruit and vegetable consumption per capita has increased steadily over the last 30 years (Fig. IA). About $45 \%$ of the fruit sold in the U.S. is consumed fresh and the remainder is processed. The proportion of fresh to processed fruit consumption remains constant although the relative components of the ratio may vary from year to year (King et al., 2000; Putnam and Allshouse, 1999). Juices, canned fruit, dried fruit and frozen fruit account for about $76 \%$, $14 \%, 8 \%$, and $2 \%$, respectively, of the processed products consumed. Per capita consumption of juices has increased over time whereas that of canned fruit has decreased. In general, per capita consumption of grape and small fruit crops has also increased over the past 30 years, in some cases dramatically (Fig. 1B-F). For example, the strawberry industry has enjoyed consistent growth in per capita consumption of fresh fruit, perhaps due to almost constant availability and com- petitive retail pricing. Increased consumption of table grapes, primarily during the period from 1980-90, may also be due in part to increased supply of affordable, off-season grapes from the southern hemisphere. Disappearance data for grape juice, raisins and wine account for per capita consumption increases in processed grapes; increases in grape concentrates as sweeteners were not considered. Per capita consumption of processed blueberries also escalated, and based on production data (USDA-NASS, 1995-2001) consumption of fresh blueberries increased as well. In contrast, per capita consumption of fresh cranberries has been static for nearly 20 years. Growth in this industry and expanded cranberry consumption are due almost entirely to the increased popularity of cranberry juice and cranberry juice blends.

Mean annual growth rates for U.S. fruit and vegetable consumption increased 8.6 and $9.3 \%$, respectively, over the decade following the development of the Food Guide Pyramid and the 5 A Day-for Better Health program (Fig. 1A-F). Similarly, fresh fruit and vegetable consumption in the United Kingdom is increasing; fresh fruit sales rose nearly $25 \%$ in 10 years whereas vegetable sales, although slightly lower than 10 years ago, have increased $4 \%$ per year since 1995 (MAFF, 1998). In the U.S., processed grapes, fresh and processed strawberries and processed caneberries have exhibited greater mean annual growth rates during the past decade than in the preceeding 20 years. In some part, these increases may have resulted from increased public awareness of the link between diet and health through national education initiatives and emerging medical information as covered by the media. Per capita consumption data therefore suggests we are well on the way to successfully improving nutritional wellbeing, prolonging health and reducing the risk of chronic diseases. But, are these per capita consumption increases really indicative of improved dietary habits within the population?

\section{Public awareness and dietary improvement}

"Although food supply data give an indication of long term trends in consumption of these [fruit and vegetable] foods, they cannot be used to infer how close the population is to meeting the health objective because the are not reported in comparable terms (i.e., servings of food ingested)" (Krebs-Smith, 1998). Recent evidence from dietary recall surveys suggested that only $20 \%$ of U.S. adults, $10 \%$ of Norwegian adults and 3\% of Scottish adults consume the recommended 5 portions $(80 \mathrm{~g})$ of fruit and vegetables daily (Cox et al., 1998, Johansson and Andersen, 1998).

Katz (1998) summarizing information from the USDA Continuing Survey of Food Intakes by Individuals (CSFII) reported that from 1995-96, the average number of servings of fruit were 1.5 and 1.4 for U.S. adult men and women, respectively. Correspondingly, only $20 \%$ of men and $24 \%$ of women consumed the recommended level of fruit. According to King et al. (2000), more than half of all Americans ingest some type of fruit or fruit drink serving nearly every day, but these consumers are eating only onehalf the amount of fruit recommended in USDA Food Guide Pyramid (less than one serving a day).

For vegetables, recent dietary recall studies reflect a greater compliance with Food Guide Pyramid and 5a-Day recommendations (Katz, 1998; Krebs-Smith, 1998), with adult men and women averaging 4.1 and 3.2 servings per day, respectively. However, nearly one-quarter of the increase in vegetables in the diet can be accounted for by an increased popularity of french fries rather than by increased levels of vegetables rich in vitamins, antioxidants and other nutrients. Moreover, only $50 \%$ of the men and $44 \%$ of the women surveyed met the recommended goals of 3 to 5 servings per day.

Improvement (or lack thereof) in the American diet after introduction of the Food Guide Pyramid is perhaps best depicted by the Healthy Eating Indices (HEI) compiled for the period 1989-90 (USDA-CNPP, 1995) and that for the period 1994-96 (Bowman et al., 1998). Based on CSFII statistics, the HEI was developed by the USDA, Center for Nutrition Policy and Promotion (CNPP) as an instrument to reflect overall dietary quality. To determine the HEI per individual, information is compiled in 10 categories by assessing how well a person's diet conforms to the Food Guide Pyramid recommendations, by evaluating 
the amount of total fat, saturated fat, cholesterol and sodium consumed and by considering variety in the diet. Scoring criteria for each category is from 0 to 10 points, with ten points indicating dietary recommendation compliance; individual criterion for scoring is adjusted for age, gender and caloric requirements.

The overall HEI scores for the populations surveyed in 1989-90 averaged 63.8 indicating that the average American diet needed improvement (Table 2 ). Only $12 \%$ of individuals received overall scores of 80 points or above indicating a good diet whereas $14 \%$ those studied earned overall scores of less than 51 points indicating a poor diet. Five years later, average overall scores had not improved and the percentage of the population categorized as having a poor diet had risen to $18 \%$. Average scores for individual components did reveal improvements over time in limiting intakes of fat and saturated fat and reducing meat consumption, in increasing intakes of grains and in improving dietary variety, but also showed declines in milk consumption as well as an increased use of salt.

In the 1989-90 report (USDACNPP, 1995), about $14 \%$ and $17 \%$ of individuals surveyed received HEI scores of 10 for fruit and vegetable consumption, respectively. By the 1994-96 study (Bowman et al., 1998) the percentage of the survey population meeting the dietary requirements of 2 to 4 servings of fruit/day had risen from $14 \%$ to $17 \%$ and 3 to 5 servings of vegetables/day had risen from $17 \%$ to $31 \%$. Although these statistics may indicate a trend toward healthier eating, data from the earlier and much larger NHANES II (1976-80) study revealed the percentages of respondents in compliance with dietary recommendations for fruit and vegetable intakes to be $29 \%$ and $27 \%$ respectively (Patterson et al., 1990). Moreover, mean HEI scores for fruit and vegetable intakes remained relatively constant since the initial data period (Table 2). Recent educational initiatives and increased understanding concerning the relationship between diet and health have changed our perception of the importance of fruit and vegetables in our diets. Apparently, however, they have not for the most part altered our dietary behavior.

There are no dietary recall studies focusing upon the intake of small fruit and grapes, so whether increased consumption is indicative of increased importance of these commodities in our diets is unknown. However, based on 1994 survey data, King et al. (2000) stated that nearly $1 / 2$ of all fruit servings were comprised of only five commodities: orange juice $=18 \%$, bananas $=9.8 \%$, fresh apples (Malus domestica) $=7.9 \%$, watermelon (Citrullus vulgaris $)=6.5 \%$, apple juice $=5.8 \%$. In contrast, Katz (1998) reported citrus, melons (Cucurbitaceae), berries and other fruit to be consumed in relatively equal proportions according to 199596 CSFII data.

In summary, we are aware that we should eat diets rich in fruit and vegetables, but many of us still fail, for various reasons, to consume these foods at current recommended levels for optimum health benefits. What then, are the factors that affect food choice, especially the preference for fruit and vegetables over other available foodstuffs?

\section{Factors influencing food choice and their effect on fruit and vegetable intakes}

According to Shepherd (1997) food choice by individuals is a complex phenomenon that is predicated upon many integrated factors. These factors may include sensory preference, physi- ological factors (pre- and postingestion), age, gender, lifestyle, behavior, personality, education, income, social pressures, marketing pressures, available product information and knowledge (labeling, media coverage, etc.), attitudes about diet and health, ethnicity and tradition, religious beliefs and social or self-identity beliefs (Abbott, 1997; Bowman et al., 1998; Cox et al., 1998; Farmakalidis, 1999; King et al., 2000; Leather, 1995; Shepherd, 1997; USDA-CNPP, 1995; Variyam et al., 1998; Wrick, 1995; Young, 1998). For each food choice event, additional external and internal inputs are considered (integrated) consciously or subconsciously by the individual, which impacts attitude, intent and behavior. Moreover, these inputs can affect a person's immediate and long term opinion about a specific food product. Based on those of others (Cardello, 1994; Imram, 1999; Shepherd, 1997) a food choice model was formulated herein to illustrate some specific barriers to the consumption of fruit (especially small fruit and grapes) and alternatively, specific opportunities for increasing their consumption (Fig. 2).

Without question, an individual's physiological status (i.e., the level of hunger or thirst felt) has a primary and immediate impact upon his or her desire to consume. Food choices also seem to be influenced by the

Table 2. Healthy Eating Index scores for study periods from 1989-90 and from 1994-96 (USDA-CNPP, 1995; Bowman et al., 1998).

\begin{tabular}{|c|c|c|}
\hline \multirow{2}{*}{$\begin{array}{l}\text { Index components } \\
\text { (standards) }^{y}\end{array}$} & \multicolumn{2}{|c|}{ Mean scores ${ }^{\mathrm{z}}$} \\
\hline & 1989-90 & 1994-96 \\
\hline Grains (6-11 servings) & 6.1 & 6.7 \\
\hline Vegetables ( $3-5$ servings $)$ & 6.1 & 6.2 \\
\hline Fruit $(2-4$ servings $)$ & 4.0 & 3.9 \\
\hline Milk (2-3 servings) & 6.7 & 5.4 \\
\hline Meat $(2-3$ servings $)$ & 7.5 & 6.5 \\
\hline Total fat (energy from fat $\leq 30 \%)$ & 6.3 & 6.8 \\
\hline Saturated fat (energy from saturated fat $<10 \%$ ) & 5.0 & 6.4 \\
\hline Cholesterol $(\leq 300 \mathrm{mg})$ & 7.9 & 7.8 \\
\hline Sodium $(\leq 2400 \mathrm{mg})$ & 7.0 & 6.3 \\
\hline Variety ( 16 different food items over $3-\mathrm{d}$ period) & 7.0 & 7.6 \\
\hline Overall score ${ }^{\mathrm{x}}$ & 63.8 & 63.6 \\
\hline
\end{tabular}

${ }^{\mathrm{z} C o m p o n e n t}$ scores for each individual ranged from $0-10$, with 10 indicating that individual meets dietary requirements. Component score means are based on individual scores of 4000, 3400, 5200, 4900, and 4800 participants in 1989, 1990, 1994, 1995, and 1996, respectively.

yStandards indicate component performance levels which receive a score of 10 . For individuals, standards are adjusted for gender and age.

${ }^{x}$ Overall scores are calculated as sums of component scores. Overall scores for each individual ranged from 0-100, with 100 indicating that the individuals diet meets all standards for healthy eating. Overall scores of less than 100 indicate the following: $80-99=$ good diet, $51-79=$ diet needs improvement, $<51=$ poor diet. In $1989-90,12 \%$, $74 \%$ and $14 \%$ of individuals studied received overal scores indicating good, needs improvement, or poor diets, respectively. In $1994-96,12 \%, 70 \%$ and $18 \%$ of individuals studied received overal scores indicating good, needs improvement, or poor diets, respectively. 


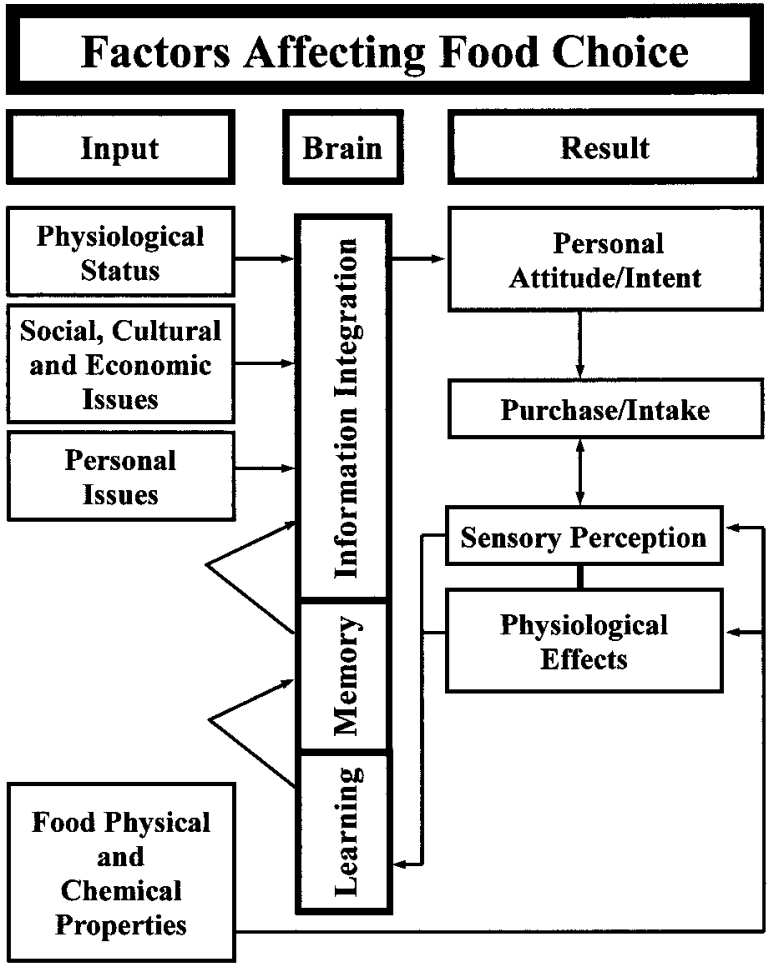

Fig. 2. Food choice model (Cardello, 1994; Imram, 1999; Shepherd, 1997).

consumer's age and gender. In both HEI studies, women received slightly higher scores than men; but males 15 to 18 years of age scored particularly low HEIs (Bowman et al., 1998; USDA-CNPP, 1995; Variyam et al., 1998). Mean HEI scores tend to be high in very young and in older individuals, and according to King et al. (2000), children under 5 years of age and adults over 70 years of age eat the most fruit. The food choice factors of age and gender may have some basis in human physiology (i.e., differences in sensory perception), but are most likely influenced either positively or negatively by social and cultural factors. For instance, higher HEI scores in women over those of men were related to increased knowledge concerning nutrients in food and a better understanding of the relationship between diet and health. Conversely, Wrick (1995) summarized work based on NHANES II data suggesting that elderly people living without a spouse, especially men are likely to have poor diets and low intakes of fruit and vegetables.

Important social, cultural and economic factors affecting fruit or fruit product consumption may include lack of advertizing/exposure, high price/ caloric unit, limited availability and limited support or encouragement from family and friends (Abbott, 1997; Anderson et al., 1998; Farmakalidis, 1999; Leather, 1995; Shepherd, 1997; Wrick, 1995). Yearly advertizing budgets for fruit and fruit products are generally several orders of magnitude less than those expended to promote carbonated beverages, fast foods, cereals, coffee and chocolate products (Leather, 1995; E. Pivonka, personal communication). The fact that fruit and vegetables are often marketed without brand names may be a factor which limits the incentive for producers and marketers to advertize.

Price/caloric unit is a major factor in food choice, especially among lower socioeconomic classes (Leather, 1995). During the period from 1995 to 1997 , per capita consumption of fruit and vegetables by the poorest $10 \%$ of the population in England was $47 \%$ and $40 \%$ below average intakes, respectively (MAFF, 1998). In the U.S., HEI scores tend to be lower than average in households with incomes that are below the poverty level. According to Bowman et al. (1998), "higher income groups have the ability to buy relatively expensive foods, such as fresh fruit and lean meats which results in better diet quality". Moreover, based on price indices, retail prices for fruit and vegetables have risen 93\% since 1982 , well above increases recorded for all food items (61\%) or all items (66\%) (Putnam and Allshouse, 1999). Percentage price increases for fresh fruit were more than double those of processed fruit. Price may remain a factor limiting fruit and vegetable consumption even when federal assistance for food procurement is available. For instance, participants in U.S. Food Stamp Program consume more meat, fat and sugar as a result of federal assistance, but their intake of fruit and vegetables remains low (Levendahl and Oliveira, 1999; Wilde et al., 2000). However, recipients of the Women
Infants and Children (WIC) Program, do benefit nutritionally from an increased intake of fruit juices supplied specifically by the program.

Issues of price may especially limit intakes of small fruit. Table 3 lists the price per serving and the price per 100 calories (419 J) of potatoes and various fresh and processed fruit commodities based on the average minimum prices found during Apr. 2001 at five supermarket chains in northeastern Ohio. Fruit and fruit product prices/serving and prices/100 calories all exceed those of potatoes. Juices were the least expensive among fruit products, but often contained added water and sweetener. Admittedly, the high prices for fresh small fruit commodities listed herein, especially raspberries and blueberries, would be lower during summer months when these commodities are in greater supply through local or even remote suppliers. However, even when small fruit commodities are in season, they tend to be priced higher than other fruit crops, perhaps reflecting limited production acreage and supply (Table 4), high shipping costs and high shelf-life losses.

Personal issues affecting fruit or fruit product consumption may include the level of knowledge concerning the products, lifestyle and available time, moral, ethical or religious concerns and self-identity (Anderson et al., 1998; Farmakalidis, 1999; Havas et al., 1998; Hilliam, 1995; Schafer et al., 1999; Shepherd, 1997; Wrick, 1995; Young, 1998). HEI scores were directly correlated with educational levels (USDA-CNPP, 1995) and "systematically related to higher knowledge of the nutrient content of foods, [and] more awareness of diet-health problems" (Variyam et al., 1998). Certainly media coverage of medical information concerning potential health benefits of specific fruit products, such as orange or cranberry juice has stimulated consumption of these items at the national level (Farmakalidis, 1999; Sloan, 2000). The health benefits derived from red wine consumption have received considerable attention within the last decade after the French Paradox was widely publicized by the press. The lower incidence of cardiovascular disease within the French population (i.e., a population that consumes relatively high levels of fat) has been attributed to the antioxi- 
dant properties of hydrocinnamic acids, flavonoids and resveratrol found predominately in grape skins (Leake, 1997). Other small fruit commodities are also gaining favorable exposure in the press as we learn more about their generally high levels of antioxidants (e.g., blueberries, Kalt et al., 2001) and of the health benefits and of specific antioxidant groups such as the ellagitannins of raspberries and strawberries (Maas et al., 1991, Xue et al., 2001). Hopefully, this increased exposure will lead to greater levels of consumption. However, health claims may also be misinterpreted or poorly understood leading to confusion and disgruntlement about healthful food products (Farmakalidis, 1999, Hilliam, 1995; Wrick, 1995; Young 1998).

Increasingly, our culture is based on convenience, which is a major factor in food choice. In a survey by the American Dietetic Association, $21 \%$ of respondents felt that healthy eating took too much time (Farmakalidis, 1999). Fresh fruit may require preparation prior to use in meals and both fresh and minimally processed fruit products have relatively short shelf lives (especially small fruit) and are perceived by consumers as requiring increased shopping time over products that are less perishable (Anderson et al., 1998; Cox et al., 1998). Moreover, in increasing frequency, meals are being eaten away from home. In these instances, the consumption of fresh fruit may be curtailed because they are generally less available than fast foods at mealtime or than other snacks from vending machines (Anderson et al., 1998; Cox et al., 1998). Even when fruit is consumed outside of the home, variety may be lacking. Apples, bananas, oranges and peaches predominate because they are easy to transport (relatively nonperishable) and may be eaten anywhere (King et al., 2000).

Safety is another personal issues that may affect food choice. Concern over pesticide residue may limit fresh fruit purchases in general (E. Pivonka, personal communication), whereas this same concern along with self-identity with environmental issues may stimulate the purchase of organically produced fruit and fruit products (Shepherd, 1997). The current controversy surrounding the safety of genetically modified organisms (GMOs) may also affect public opinion about the safety of fruit in the diet, if and when new cultivars derived through these techniques are released. According to the National Science Board (2000), overall public opinion regarding the safety of GMOs has changed little in the last 10 years, but concern about the potential risks of this technology has increased dramatically among well-educated individuals. Conversely, self-identity issues, such as the self-esteem gained through consuming a diet which is good for you, may positively influence intake of fruit and vegetables (Havas et al., 1998; Schafer et al., 1999).

Finally, through learning and memory, past food experiences are also considered by individuals making food choices. In other words, a food's physical and chemical properties as they influence sensory attributes (texture, mouthfeel, color, taste, aroma, flavor) and the immediate and long term feeling of physiological well-being, directly affect immediate and future consumption (Shepherd, 1997). Quality, especially flavor quality is a key issue:

Table 3. Mean fruit and vegetable commodity prices at five supermarkets in northeastern Ohio during April 2001. ${ }^{\mathrm{z}}$

\begin{tabular}{|c|c|c|c|c|}
\hline Commodity & $\begin{array}{l}\text { Serving } \\
\text { size }^{y, x}\end{array}$ & $\begin{array}{l}\text { Calories/ } \\
\text { serving }^{\mathrm{y}, \mathrm{w}}\end{array}$ & $\begin{array}{c}\text { Price/ } \\
\text { serving }(\$)\end{array}$ & $\begin{array}{c}\text { Price/100 } \\
\text { calories }^{\mathrm{w}}(\$)\end{array}$ \\
\hline \multicolumn{5}{|l|}{ Fresh fruit and vegetables } \\
\hline Bananas & 126 & 110 & $0.13 \pm 0.02$ & $0.12 \pm 0.02$ \\
\hline Navel oranges & 154 & 70 & $0.23 \pm 0.01$ & $0.33 \pm 0.01$ \\
\hline Apples & 154 & 80 & $0.35 \pm 0.05$ & $0.43 \pm 0.06$ \\
\hline Raspberries & 125 & 58 & $2.92 \pm 0.29$ & $5.30 \pm 0.80$ \\
\hline Blueberries & 140 & 100 & $5.22 \pm 0.37$ & $5.22 \pm 0.37$ \\
\hline \multicolumn{5}{|l|}{ Frozen fruit (whole) } \\
\hline Strawberries & 148 & 50 & $0.66 \pm 0.08$ & $1.31 \pm 0.16$ \\
\hline Raspberries & 165 & 75 & $1.00 \pm 0.06$ & $1.30 \pm 0.11$ \\
\hline \multicolumn{5}{|l|}{ Fruit juices } \\
\hline Orange juice (refrigerated) & 237 & 110 & $0.23 \pm 0.02$ & $0.21 \pm 0.02$ \\
\hline Orange juice (frozen) & 237 & 120 & $0.19 \pm 0.02$ & $0.16 \pm 0.02$ \\
\hline Grape juice (bottled) & 237 & 154 & $0.33 \pm 0.03$ & $0.21 \pm 0.02$ \\
\hline Grape juice (frozen) & 237 & 144 & $0.23 \pm 0.02$ & $0.17 \pm 0.03$ \\
\hline Cranberry juice (bottled) & 237 & 136 & $0.26 \pm 0.02$ & $0.19 \pm 0.02$ \\
\hline
\end{tabular}

${ }^{z}$ Supermarkets included three nationally or regionally recognized chains and two locally-owned chains. Prices and additional information from nutrition labeling were recorded from the lowest-priced products available.

yBased on the tabular data of Van Duyn, 1999 for fresh products and on nutritional labeling information for packaged products.

${ }^{x}$ For fresh frozen and dried products, serving sizes are reported in grams $(1.0 \mathrm{oz}=28.35 \mathrm{~g})$; for liquid products serving sizes are reported in milliliters $(1.0 \mathrm{fl} \mathrm{oz}=29.6 \mathrm{~mL})$.

w 100 calories $=419 \mathrm{~J}$.

vSolanum tuberosum. 
Table 4. Year 2000 production statistics for potato, apples and various small fruit crops (USDA-NASS, 1995-2001).

\begin{tabular}{lccr}
\hline Commodity & $\begin{array}{c}\text { Bearing } \\
\text { acreage } \\
(\mathbf{1 , 0 0 0} \text { acres })^{\mathbf{z}}\end{array}$ & $\begin{array}{c}\text { Utilized } \\
\text { production } \\
(\mathbf{1 0 0 0} \text { tons })^{\mathbf{y}}\end{array}$ & $\begin{array}{c}\text { Value of } \\
\text { utilized } \\
\text { production } \\
\mathbf{( \$ 1 0 0 0 )}\end{array}$ \\
\hline Potatoes & $1,351.6$ & $25,798.2$ & $2,539,561$ \\
Apples & 451.3 & $5,167.4$ & $1,553,536$ \\
Blueberries & 40.3 & 90.8 & 176,571 \\
Caneberries & 23.5 & 82.8 & 85,200 \\
Cranberries & 36.6 & 264.4 & 112,235 \\
Grapes & 956.5 & $7,314.6$ & $3,063,918$ \\
Strawberries & 47.7 & 923.8 & $1,013,537$ \\
\hline
\end{tabular}

${ }^{\mathrm{z}} 1.0$ acre $=0.405$ ha.

${ }^{\mathrm{y}} 1.0$ ton $=0.907 \mathrm{t}$

“...Health claims alone did not guarantee success of a functional food. Other major interacting factors included quality - most importantly flavor" (Hilliam, 1995)

“...Given the choice, many consumers will select the 'healthy' product over the standard, provided there is no taste compromise..." (Farmakalidis, 1999) “...While consumers continue to want food with lower fat and cholesterol, they've made it clear that they want taste back on the front burner..." (Sloan, 1993).

When consumers were asked about their criterion for purchasing food products, $78 \%$ to $79 \%$ indicated nutritional content to be an important consideration, whereas $88 \%$ to $91 \%$ listed flavor as a determining factor (Farmakalidis, 1999; E. Pivonka, personal communication). Small fruit have long been appreciated for their delicate and unique flavors (Bowling, 2000 ) and serving them is often considered to be a treat for family and guests. Because of this, and because of their relatively high price, it is important for producers to consistently offer small fruit products to the consumer that are of the highest quality. Real or perceived quality shortfalls can greatly impact a person's desire to eat fresh fruit (E. Pivonka, personal communication).

\section{Outlook for increased small fruit consumption}

What are the factors which favor the consumption of small fruit and grapes as part of a well-rounded diet? Certainly, they are rich in phytochemicals (antioxidants and others) that are now recognized by the consuming public as being beneficial to health and longevity. However, as

the discussion above suggests, the greatest incentives for consumption of small fruit lies with their unique and highly pleasurable sensory qualities and with their image as highly desirable (dessert-like) commodities. Interestingly, this notion of small fruit as treats may also deter consumers to some extent from routinely including them in their daily diets. Other deterrents to small fruit consumption may be of greater significance such as their high cost/serving or cost/calorie and substantial seasonal fluctuation in price, which may exclude many people from lower or middle socioeconomic ranks from routinely purchasing them. The relative perishability (short storage or shelf life) of most small fruit commodities contributes to their high cost, but also makes them difficult to transport without loss in quality for producers, wholesalers, marketers and consumers alike. The seasonality of some small fruit and the limited acreage devoted to small fruit production may also exacerbate high prices and curtail increased consumption by consumers.

However, there are several strategies that might be successfully employed to capitalize on the sensory and nutritional qualities of small fruit and to overcome barriers related to price and perishability. For instance, at the direct market level, King et al. (2000) suggests the development of innovative marketing strategies (e.g., those which involve product variety, education and entertainment) as a means to promote sales of fruit in general. On a broader level, state and regional marketing programs (e.g., OHIO PROUD ${ }^{\circ}$, the signature marketing program of the Ohio Department of Agriculture, Reynoldsburg) might increase their promotional activities with respect to the desirability and health benefits of increased consumption of locally produced small fruit and small fruit products. National or international commodity groups (e.g., The North American Blueberry Council, Folsom, Calif.) may do likewise on a much larger scale. Increased incentives and improved technology for the global marketing of these commodities might help insure consistent supplies and stabilize prices at the local level. Increases in fruit consumption in general and in small fruit in particular would undoubtedly be aided by continued efforts of local and national programs to increase public knowledge and understanding of the health benefits of these commodities. In turn, these health benefits might be clarified through expanded research efforts to further explore phytochemical content within small fruit, to delineate the parameters that affect phytochemical content and availability, to understand the physiological mechanisms by which they intercede in the progress of chronic disease and, if necessary to develop fruit-based, cost-effective, and physiologically active nutriceutical products for ease of inclusion in the diet.

Moreover, because of the paramount importance of small fruit quality in motivating consumption, genetic, pre- and postharvest physiological and cultural research focused upon and resulting in quality improvement might also bring about increases in small fruit intakes. For instance, the development of packaging systems specifically designed to deliver products from vending machines or for use in lunch boxes may increase opportunities to consume fresh small fruit at the workplace. Physiological and genetic research elucidating mechanisms for coordinated control of the ripening processes in small fruit might aid in the development of cultivars that bear fruit that ripen fully even though harvested prior to maturity. Similar lines of research may result in fruit with improved shippability and longer shelf life. Finally, genetic research exploring the inheritance of the components of quality and breeding efforts to maximize them may result in cultivars that consistently produce fruit of superior sensory quality.

Finally, what if we succeed in substantially increasing the demand for and consumption of small fruit and small fruit products? Obviously, if de- 
mand increases, supply must increase as well through increased production. Current production statistics for potato, apple and small fruit crops are listed in Table 4 . It can be easily concluded from these data that small fruit crops (excluding grapes, perhaps) are produced on relatively limited acreage, that they are in limited supply and that the value of fruit produced is very high (e.g., strawberry acreage and utilized production are only $3.5 \%$ as great as those of potato, but the resulting crop is worth about $40 \%$ as much as potato utilized production). Since product availability often affects production profitability, growth within this industry would have to be wellcoordinated in order to maintain adequate profit margins for producers while hopefully, providing consumers with a more affordable product. Even so, availability of suitable land for expanded production may be limiting, as well as the infrastructure necessary to produce, harvest, ship, store and market a greatly increased volume of these commodities.

Despite the barriers to increasing small fruit consumption, it remains a laudable goal. As many as 1.4 million deaths per year in the U.S. are attributable to cancer, diabetes, heart disease and stroke and these and other dietrelated diseases cost society an estimate $\$ 250$ billion each year in medical costs and lost productivity (Bowman et al., 1998). Adopting diets that contain adequate levels of fruit and vegetables would obviously improve these statistics. In fact, according to Pivonka (E. Pivonka, personal communication) and Krebs-Smith (1998), the current recommended 2 to 4 servings of fruit and 3 to 5 servings of vegetables, only represent minimums, which, if exceeded, could still offer greater health benefits. Phytochemical-rich small fruit have a definite place in these efforts.

\section{Literature cited}

Abbott, R. 1997. Food and nutrition information: A study of sources, uses and understanding. British Food J. 99:43-49.

Achterberg, C. 1994. How to put the food guide pyramid into practice. J. Amer. Dietetic Assn. 94:1030-1035.

Anderson, A.S., D.N. Cox, S. McKellar, J. Reynolds, M.E.J. Lean, and D.J. Mela. 1998. Take five, a nutrition education intervention to increase fruit and vegetable intakes: Impact on attitudes towards di- etary change. British J. Nutr. 80:133-140.

Balch, G.I., K. Loughrey, L Weinberg, D. Lurie, and E. Eisner. 1997. Probing consumer benefits and barriers for the national 5 a day campaign: Focus group findings. J. Nutr. Educ. 29:178-183.

Bowling, B.L. 2000. The berry growers companion. Timber Press, Portland, Ore.

Bowman, S.A., M. Lino, S.A. Gerrior, and P.P. Basiotis. 1998. The healthy eating index 1994-1996. USDA Ctr. Nutr. Policy Promotion Publ. CNPP-5, Wash., D.C.

Cardello, A.V. 1994. Consumer expectations and their role in food acceptance, $\mathrm{p}$. 253-297. In: H.J.H. McFie and D.M.H Thomson (eds.). Measurement of food preferences. Blackie Academic and Professional, Glasgow.

Chapman, N. 1999. The new great pyramid... of USDA. Prepared Foods 168:28.

Cox, D.N., A.S. Anderson, J. Reynolds, S. McKellar, M.E.J. Lean, and D.J. Mela. 1998. Take five, a nutrition education intervention to increase fruit and vegetable intakes: Impact on consumer choice and nutrient intakes. British J. Nutr. 80:123131.

Creasy, L.L. and M.T. Creasy. 1998. Grape chemistry and the significance of resveratrol: An overview. Pharmaceutical Biol. 36(Suppl.):8-13.

Farmakalidis, E. 1999. Consumer issues in relation to antioxidants, p. 423-435. In: N.J. Temple and M.L. Garg (eds.). Antioxidants in human health and disease. CABI Publ., New York.

Food Technology Editorial Staff. 1992. Food guide pyramid replaced the basic 4 circle. Food Technol. 46:64-67.

Häkkinen, S.H., S.O. Kärenlampi, I.M. Heinonen, H.M. Mykkänen, and A.R. Törrönen. 1999. Content of the flavonols quercetin, myricetin and kaempferol in 25 edible berries. J. Agr. Food Chem. 47:2274-2279.

Havas, S., K. Treiman, P. Langenberg, M. Ballesteros, J. Anliker, D. Damron, and R. Feldman. 1998. Factors associated with fruit and vegetable consumption among women participating in WIC. J. Amer. Dietetic Assn. 98:1141-1148.

Hertog, M.G.L., G. Van Poppel, and D. Verhoeven. 1997. Potentially anticarcinogenic secondary metabolites from fruits and vegetables, p. 313-329. In: F.A. Tomás-Barberán and R.J. Robins (eds.). Phytochemistry of fruit and vegetables. Clarendon Press, Oxford.

Hilliam, M. 1995. Functional foods: The western consumer viewpoint. Nutr. Rev.

\section{4(11):S189-S194.}

IFIC. 1999. Functional foods background. Intl. Food Info. Council Foundation, Wash., D.C.

Imram, N. 1999. The role of visual cues in consumer perception and acceptance of a food product. Nutr. Food Sci. 5:224-228.

Jang, M., and J.M. Pezzuto. 1999. Cancer chemopreventive activity of resveratrol. Drugs Under Expt. Clinical Res. 25:6577.

Johansson, L. and L.F. Andersen. 1998. Who eats 5 a day? Intake of fruits and vegetables among Norwegians in relation to gender and lifestyle. J. Amer. Dietetic Assn. 98:689-691.

Kalt, W., A. Howell, C.F. Forney, and J.E. McDonald. 2001. Horticultural factors affecting antioxidant capacity of blueberries and other small fruit. HortTechnology 11(4)523-528.

Katz, F. 1998. USDA surveys show what Americans eat. Food Technol. 52:50-54.

King, B.S., J.L Tietyen, and S.S. Vickner. 2000. Food and agriculture: Consumer trends and opportunities-Fruits. Univ. Ky. Coop. Ext. Publ. IP-58D.

Krebs-Smith, S.M. 1998. Progress in improving diet to reduce cancer risk. Cancer 83:1425-1432.

Leake, D.S. 1997. The possible role of antioxidants in fruits and vegetables in protecting against coronary heart disease, p. 287-311. In: F.A. Tomás-Barberán and R.J. Robins (eds.). Phytochemistry of fruit and vegetables. Clarendon Press, Oxford.

Leather, S. 1995. Fruit and vegetables: Consumption patterns and health consequences. British Food J. 97(7):10-17.

Levedahl, J.W. and V. Oliveira. 1999. Dietary impacts of food assistance programs, p. 307-330. In: E. Frazão (ed.). America's eating habits: changes and consequences. USDA-Econ. Res. Serv. Agr. Info. Bul. 750, Wash., D.C.

Maas, J.L., G.J. Galletta and G.D. Stoner. 1991. Ellagic acid, an anticarcinogen in fruits, especially in strawberries-A review. HortScience 26:10-14.

MAFF. 1998. Fruity Brits opt for healthier lifestyle. News Release 436/98, Ministry of Agr., Fisheries and Food, London, U.K.

National Science Board. 2000. Science and engineering indicators-2000. Natl. Sci. Foundation Publ. NSB-00-1, Arlington, Va.

Patterson, B.H., G. Block, W.F. Rosenberger, D. Pee, and L.L. Kahle. 1990. Fruit and vegetables in the American diet: Data from the NHANES II survey. Amer. 
J. Public Health 80:1443-1449.

Putnam, J.J. and J.E. Allshouse. 1999. Food consumption, prices and expenditures, 1970-1997. USDA-Econ. Res. Serv. Stat. Bul. 965, Wash., D.C.

Rommel, A. and R.E. Wrolstad. 1993. Composition of flavonols in red raspberry juice as influenced by cultivar, processing and environmental factors. J. Agr. Food Chem. 41:1941-1950.

Scarlata, C.J. and S.E. Ebeler. 1999. Vitamins, especially flavonoids in common beverages are powerful in vitro antioxidants which enrich lower density lipoproteins and increase their oxidative resistance after ex vivo spiking in human plasma. J. Agr. Food Chem. 47:2502-2504.

Schafer, E., R.B. Schafer, P.M. Keith, and J. Bose. 1999. Self-esteem and fruit and vegetable intake in women and men. J. Nutr. Educ. 31:153-160.

Shepherd, R. 1997. The psychology of food choice: The influence of attitudes and flavour perception, p. 157-172. In: F.A. Tomás-Barberán and R.J. Robins (eds.). Phytochemistry of fruit and vegetables. Clarendon Press, Oxford.

Shahidi, F. and M. Naczk. 1995. Food phenolics: An overview, p. 1-8. In: Food phenolics: Sources, chemistry, effects, applications. Technomic Publ. Co., Lancaster, $\mathrm{Pa}$

Sloan, A.E. 1993. Tastier, healthier, and down right more fun to eat! Food Technol. $47(9): 138,140$.

Sloan, A.E. 2000. Fortification frenzy: the new wellness mindset. Food Technol. 54(2):20.

Steinmetz, K.A. and J.D. Potter. 1996. Vegetables, fruit and cancer prevention: A review. J. Amer. Dietetic Assn. 96:10271039.
USDA-ARS. 1999. Can foods forestall aging. Agr. Res. 47:15-17 (Feb.).

USDA-CNPP. 1995. The healthy eating index. USDA Center for Nutrition Policy and Promotion Publ. CNPP-1, Wash., D.C.

USDA-NASS. 1995-2001. Annual summary reports for non-citrus fruits and nuts, vegetables and crop production. National Statistics Board, Wash., D.C.

Van Duyn, M.A.S. 1999. Year 2000 dietary guidelines: The case for fruits and vegetables first. Produce for Better Health Foundation, Wilmington, Del.

Variyam, J.N., J. Blaylock, and D. Smallwood. 1998. USDA's healthy eating index and nutrition information. USDA Center for Nutrition Policy and Promotion Tech. Bul. 1866, Wash., D.C.

Wang, H., G. Cao, and R.L. Prior. 1996. Total antioxidant capacity of fruits. J. Agricultural Food Chem. 44:701-705.

Wilde, P.E., P.E. McNamara, and C.K. Ranney. 2000. The effect on dietary quality of participation in the food stamp and WIC programs. USDA-Economic Research Service Food Assistance and Nutr. Res. Rpt. 9, Washington D.C.

Wrick, K.L. 1995. Consumer issues and expectations for functional foods. Critical Rev. Food Sci. Nutr. 35:167-173.

Xue, H.W., R.M. Aziz, N.J. Sun, J.M. Cassady, L.M. Kamendulis, Y. Xu, G.D. Stoner, and J.E. Klaunig. 2001. Inhibition of cellular transformation by berry extracts. Carcinogenesis 22:351-356.

Young. J. 1998. Consumer issues and functional foods, p. 167-173. In: M.J. Sadler and M. Saltmarsh (eds.). Functional foods, the consumer, the products and the evidence. The Royal Society of Chemistry, Cambridge UK. 